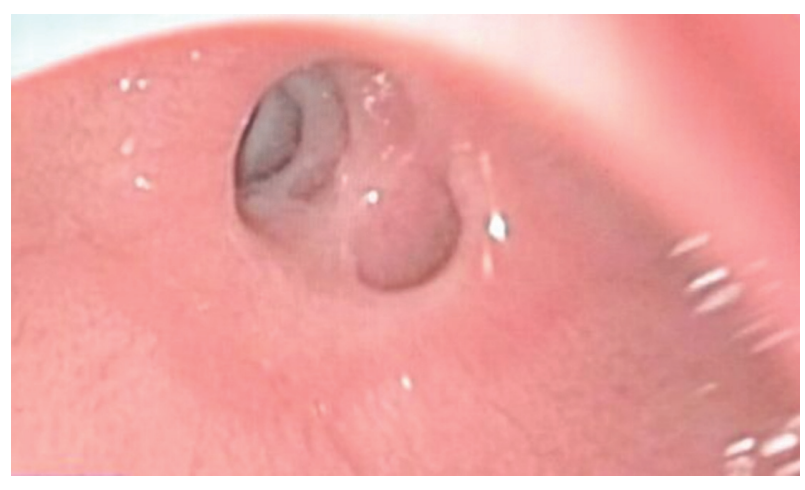

Abstract IDDF2021-ABS-0035 Figure 2

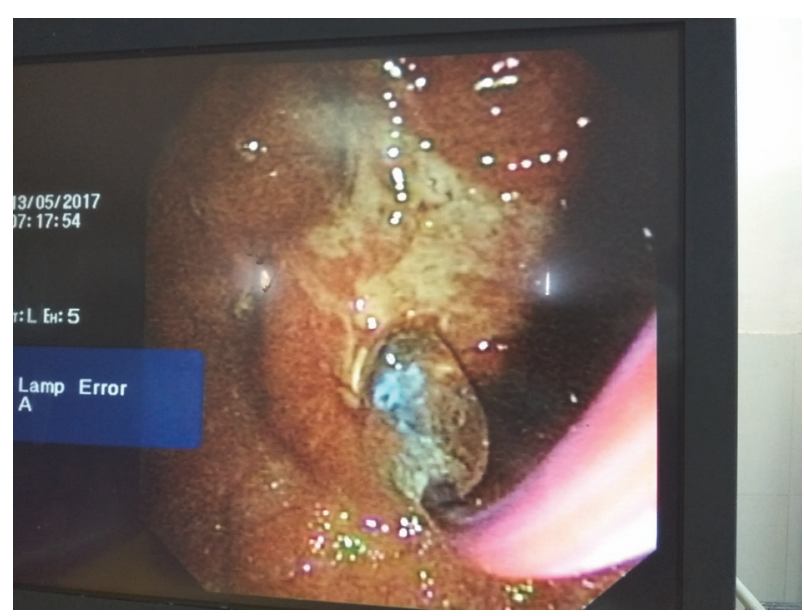

Abstract IDDF2021-ABS-0035 Figure 3

part of duodenum). The mean number of opium-containing analgesic tablets per patient was $5.2 \pm 3.1$ per day for duration of $33.1 \pm 25.8$ months. Vomiting (96.1\%), abdominal pain (93.5\%) and weight loss (85.2\%) were the most common presenting features. Forty-three $(71.6 \%)$ patients improved with endotherapy along with strict opium abstinence as shown in figure 3 while $17(28.3 \%)$ patients required surgical treatment (IDDF2021-ABS-0035 Figure 3. Endoscopic dilatation of pyloric stricture).

Conclusions Opium addiction is also a cause of benign gastric outlet obstruction. Gut involvement is seen on multiple sites, with most common at the junction of the first and second part of the duodenum. A successful outcome of endotherapy is more likely in these patients.

\section{IDDF2021-ABS-0036 PROGNOSTIC VALUE OF THE CEA, RDW- CV, RDW-SD COMBINED WITH CIP2A IN THE SERUM OF PATIENTS WITH COLORECTAL CANCER}

${ }^{1}$ Wei Chen*, ${ }^{2}$ Xiang Peng. 'Department of Colorectal Surgery, Guangdong Provincial Key Laboratory of Colorectal and Pelvic Floor Disease, The Sixth Affiliated Hospital, Sun Yat-sen University, China; 'Gastroenterology, Guangdong Provincial Key Laboratory of Colorectal and Pelvic Floor Disease, The Sixth Affiliated Hospital, Sun Yat-sen University, China

10.1136/gutjnl-2021-IDDF.114
Background To investigate the prognostic value of CEA, RDW-CV, RDW-SD and CIP2A in the serum of colorectal cancer (CRC) patients after resection.

Methods The clinical and follow-up data of 61 CRC patients who underwent surgery in the sixth Affiliated Hospital of Sun Yat-sen University January 2012 and December. The association between the CIP2A, CEA, RDW-CV, RDW-SD and the prognosis was evaluated. The Kaplan-Meier curves were used to identify the overall survival (OS) rates of the different groups.

Results At the end of the follow-up period (2020), the median follow-up is 207 months (range, 17-260 months). The 5-year OS of patients with low level of serum CIP2A was not better than that of high level, which is contrary to other indicators (CEA, RDW-CV and RDW-SD). Cox multivariate analysis showed that the age, $\mathrm{N}$ stage and $\mathrm{M}$ stage were independent factors influencing patient survival. The CRRC (combination of CEA, RDW-CV, RDW-SD and CIP2A) has better prognostic power than other indicators (CIP2A, CEA, RDW-CV and RDW-SD) according to the receiver operating characteristic (ROC) curve analysis.

Conclusions CRRC could be used as a better prognostic biomarker for CRC than the CEA, RDW-CV, RDW-SD and CIP2A alone.

\section{IDDF2021-ABS-0037 PROSPECTIVE EVALUATION OF AMOXICILLIN-CLARITHROMYCIN TRIPLE THERAPY IN PRIVATE CLINICAL PRACTICE IN HONG KONG FROM 2008 TO 2020}

Natalie Ning Yan Choi*, Sabrina Zi Yi Tee. Barts and the London, UK

\subsection{6/gutjnl-2021-IDDF.115}

Background There is a scarcity of local data regarding the current effectiveness of standard triple therapy in the treatment of Heliobacter pylori infection. To address the rising trend of bacterial resistance to antibiotics, we aim to monitor the efficacy of clarithromycin-containing triple therapy as a first-line treatment in Hong Kong and to assess the need for a longer treatment duration.

Methods 440 patients with a positive rapid urease test in oesophago-gastro-duodenoscopy (OGD) received clarithromycin-based triple therapy for a period of 7, 10 or 14 days according to treatment guidelines in the corresponding period of time. Patient demographics, indications for endoscopy and endoscopic diagnoses are detailed in Table 1 (IDDF2021-ABS0037 Table 1). Either a 7-day or 10-day treatment course was given between the period of 2008-2013, while a 10-day course was given from 2014-2015 and a 14-day course was given from 2016 onwards. Data analysis was carried out with chi-squared tests.

Data presented as $\mathrm{n}(\%)$. Both diagnoses were counted for those with two diagnosis, thus percentage total may exceed $100 \%$. Other indications for endoscopy included anorexia, epigastric discomfort, globus in throat, odynophagia, weight loss, dysphagia, hiccup, raised CA72.4, raised CEA, tarry stool, and those who were asymptomatic. Other endoscopic diagnoses included oesophageal ulcer, oesophagitis, gastric polyp, duodenitis, and oesophageal squamous dysplasia. 